\section{Patient experiences with oral mucositis caused by chemo-/radiotherapy: a critical qualitative literature review}

\section{Daniela Zanolin, ${ }^{1}$ Christine Widmer, ${ }^{2}$ Eva-Maria Panfil ${ }^{3}$}

${ }^{1}$ Vorarlberg Institute for Vascular Investigation and Treatment, Hospital Feldkirch, Feldkirch, Austria; ${ }^{2}$ Canton Hospital of St. Gallen, Department of Oncology, St. Gallen, Switzerland 3University of Applied Sciences, Bern, Switzerland

\begin{abstract}
Mucositis is one of the most common side effects of chemotherapy and radiotherapy. In order to develop an evidence-based clinical practice guideline for the management of tumor therapy-induced-oral mucositis, it was necessary to capture the patients' perspective. Therefore the aim of this critical literature review was to explore the experience of patients with therapy-induced-oral mucositis. Searches were carried out using a systematic search strategy in CINAHL and Medline. Qualitative studies investigating the view of cancer patients receiving chemotherapy and/or radiotherapy and suffering from oral mucositis were included. Study selection and quality assessment were performed by two independent reviewers. Only two qualitative studies ( $\mathrm{n}=28$ patients) met the inclusion criteria. Oral mucositis caused by cancer therapy is associated with serious complications concerning pain, eating, swallowing, speaking, sadness, lack of energy, and distress. Mucositis was described as the worst side effect of cancer therapy. Providing patient-centered care requires understanding the experience and the needs of patients and their families. Mucositis is associated with complex physical, psychological and social consequences. Unfortunately, both studies were performed in different cultural backgrounds and health care systems, so the results cannot simply be transferred to German-speaking countries. Further research is needed to gain a deeper understanding of living with mucositis.
\end{abstract}

\section{Introduction}

Chemotherapy and radiotherapy are associated with many side effects. Mucositis is regarded as one of the most common side effects of chemotherapy and radiotherapy. ${ }^{1}$ Mucosal lesions of various severity may cause pain in mouth and throat area, induce problems with food intake, limit patient's quality of life and have economic consequences. Cancer patients with oral mucositis experience more pain, often receive parenteral nutrition, require more frequent consultations and higher emergency costs and are hospitalized longer than patients without mucositis., ${ }^{2,3}$ Furthermore, mucositis may even become so intense that tumor therapy has to be discontinued for a period of time. ${ }^{4,5}$ These problems present a major challenge for tumor treatment. With symptoms of mucositis and an increased risk of infection, patients need more staff resources and cause higher treatment costs. To meet these challenges, guidelines, studies and reports about interventions for prevention or minimization of mucositis are required. ${ }^{6}$ However, the meaning of mucositis for cancer patients is rarely addressed.

In Switzerland there is no national strategy at the federal level that prescribes the development of guidelines in general. Ensuring the supply mandate is largely the responsibility of the 26 cantons in Switzerland (7 million inhabitants). This results in 26 different health care systems, which have only a few interfaces. Therefore, it is it difficult to ensure a common national policy strategy for health care needs. The development of guidelines is inconsistent and often does not correspond to scientific standards. There is a lack of national evidence-based nursing guidelines which could give recommendations for health care facilities in Switzerland. To meet the needs for evidence-based guidelines, the Oncology Network Eastern Switzerland in collaboration with the Swiss Centre for Evidence-Based Nursing intends to develop guidelines for oncology care on a consistent, high quality level ${ }^{7}$ but with limited financial resources. The methods are oriented on internationally established rules for the development and adapting of guidelines. ${ }^{8}$ Requirements are the identification of patient-important outcomes and the ranking of the relative importance of theses outcomes. Therefore, the purpose of this study was to examine patients' experience with oral mucositis.

\section{Methods of Research}

A critical literature review was conducted with the aim to provide an evidence-based summary of findings regarding patients' experience with oral mucositis. Searches were carried out using a systematic search strategy in Medline and CINAHL in January 2014. Qualitative studies with cancer patients receiving chemothera-
Correspondence: Eva-Maria Panfil, University of Applied Sciences, Murtenstrasse 10, 3008 Bern, Switzerland.

Tel.: +41.31.848.35.59.

E-mail: eva-maria@panfil.eu

Key words: cancer, neoplasm, oral mucositis, experiences, review.

Received for publication: 9 April 2014.

Revision received: 24 June 2014.

Accepted for publication: 24 June 2014.

This work is licensed under a Creative Commons Attribution NonCommercial 3.0 License (CC BYNC 3.0).

CCopyright D. Zanolin et al., 2014

Licensee PAGEPress, Italy

Nursing Reports 2014; 4:3647

doi:10.4081/nursrep.2014.3647

py and/or radiotherapy and suffering from oral mucositis were included, independent of cancer type and of the degree of mucositis. Only studies in German or English language were considered. Sensitive search strategies (Table 1) were developed for each database using filters to identify qualitative research., ${ }^{9,10}$ There were no restrictions/limits concerning age, date of study or publication date. The reference lists of included papers were checked for further studies. The literature search was performed by two authors (DZ, EP). Titles and abstracts of all studies identified were examined independently by two review authors (DZ, EP). If studies appeared to meet the inclusion criteria or did not allow a clear decision on the basis of title and abstract, the full report was checked. Disagreements were resolved by discussion. All excluded studies were recorded, mentioning specified reasons for exclusion.

Data extraction (research question, design, sample, setting, data collection, results) and assessment of the methodological quality were carried out by two authors (DZ, EP) independently using the tool of Behrens and Langer. ${ }^{11}$ This tool addresses eleven specific domains, namely research question, design, literature search, selection of participants, description of participants, description of the researcher, data collection, data analysis, data saturation, presentation of results, and validation of results. Disagreements were resolved by discussion. Each of the questions were answered with Yes, No or Don't know. In case of missing data, the authors were contacted. The results of the methodological quality assessment were presented graphically using Review Manager 5 of the Cochrane Collaboration. The findings of the studies were summarized without using a special thematic synthesis ${ }^{12}$ due to the heterogeneity of the studies. 


\section{Results}

A total of 92 studies were identified (Figure 1). After reviewing titles and abstracts, 14 studies were identified as potentially relevant. ${ }^{13-26}$

Reasons for exclusion (Table 2) ) $^{13-26}$ were quantitative study design and inadequate focus. Finally two studies ${ }^{27,28}$ were included. The studies were conducted in Australia ${ }^{28}$ and Hong Kong. ${ }^{27}$

\section{Quality assessment}

Both authors were contacted to obtain missing information.

Study quality of both studies was generally good (Figure 2). The study of Borbasi et al. ${ }^{28}$ showed no insufficiency, whereas Cheng's study ${ }^{27}$ had minor deficiencies in study quality. Only one item description of the researcher was assessed with unclear, due to indistinct available data in the publication as well as insufficient information from the author.

\section{Description of studies}

Cheng $^{27}$ conducted semi-structured in-depth interviews with 22 children (male $=10$, mean age 12 years) who had developed oral mucositis during chemotherapy and were treated at a cancer center in Hong Kong (Table 3). Furthermore, one parent of each family was also interviewed about experience with oral mucositis during chemotherapy (female $=21$, mean age 41 years). Using qualitative content analysis the authors identified five categories: symptom experience, negative emotional outcomes, the dilemma of eating, challenges in oral care, health care needs.

Children and their parents described oral mucositis as the worst side effect of chemotherapy, by far exceeding nausea and vomiting. Symptom experience, especially severe pain and swelling, can be called a constant companion with serious complications concerning eating, speaking, swallowing, and pain. Children retired from social life because of great pain and lack of energy/lethargy. The correlation between symptoms and corresponding reactions is caused by great distress of children and parents. Children express stressful experiences especially in forms of sadness, anxiety and crying.

Mucositis-associated eating and drinking problems were a great challenge in the daily life of children and their parents. Both experienced the dilemma between hunger, pain, inability of food intake and knowledge about the importance of nutrition for improving health status. Parents reported great distress, having to force their children to eat and drink. Parenteral nutrition was not perceived as a positive solution. Oral care caused the same problems and tensions as food intake due to severe feelings of pain. Unexpected and unprepared by professionals, children suffered from severe mucositis (grade 3 and 4 according to WHO classification). Therefore parents indicated the need for more information about the disease process of oral mucositis, risks, complications and food selection. They also expressed their wish for better mental preparation. Children needed more emotional support from professionals during oral mucositis. Moreover children and parents have criticized the lack of pain management.

Borbasi et al. ${ }^{28}$ described similar issues in their phenomenological study (Table 3). They carried out in-depth interviews with six adult patients (male $=4$, mean age 51.5 years) suffering from cytotoxic therapy in conjunction with allogeneic hematopoietic stem cell transplantation. Study participants were recruited from a special clinic for cancer patients in South Australia. These patients experienced mucositis as a linear process consisting of three phases: preparatory, peak and persisting phase. Five categories were identified: presence of nurses, therapeutic interventions, manifestation of mucositis, distress with regard to eating and the value of treatment No pain, no gain. Important for participants was support of nursing staff during oral mucositis. Especially in seemingly hopeless situations the nurses' $s$ support and counseling was perceived as valuable and important. Study participants emphasized how important it was for them that nurses respected their wishes and needs with regard to treatment options. Therapeutic interventions for oral care were followed strictly, if physically possible. Participants reported manifestations of mucositis in mouth, pharynx, esophagus and stomach, which was leading to huge limitations in food intake, requiring parenteral nutrition. The experience of painful or impossible food intake made aware the importance of food for life. Mucositis was described as the worst side effect of cancer therapy and was seen as a part of cancer therapy which cannot be prevented to become healthy again.

\section{Discussion}

The purpose of this review was to explore patients' experience of oral mucositis as a side effect of chemo-/radiotherapy. There are a number of intervention trials and reviews about mucositis, for example the use of amifostine for the prevention and treatment of oral mucositis in cancer patients. ${ }^{29}$ Even if it is known, that mucositis is distressing, this study adds based on a systematic review, that there is limited

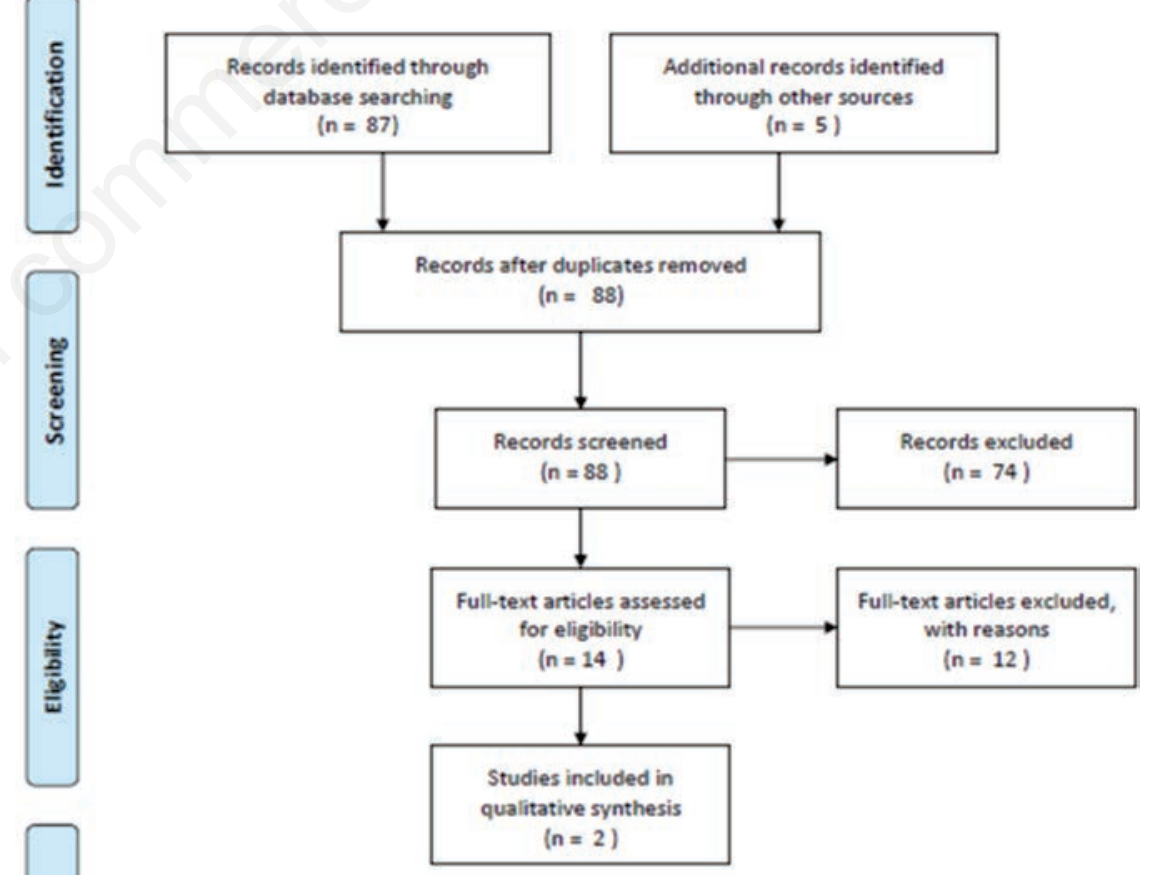

Figure 1. Flow chart of the selection process. 
research regarding patients' experiences. Due to the defined inclusion- and exclusion criteria, a total of only two studies with 28 participants could be included. The term qualitative and the use of only two databases might have restricted our search. We aimed to explore the meaning that the patients hold about living with a chemo-/radiotherapy caused mucositis. Mixed and quantitative research designs were excluded because we were not interested in the extent of burden.

Both studies were of high methodological quality. A synthesis of the findings was not performed due to the limited number of studies, different and small samples. Therefore, only an approximate idea can be conveyed about the meaning of mucositis for those affected by it.

As results indicate, oral mucositis caused by cancer therapy is associated with complex physical, psychological and social effects, and leads to significant restrictions in daily life and well-

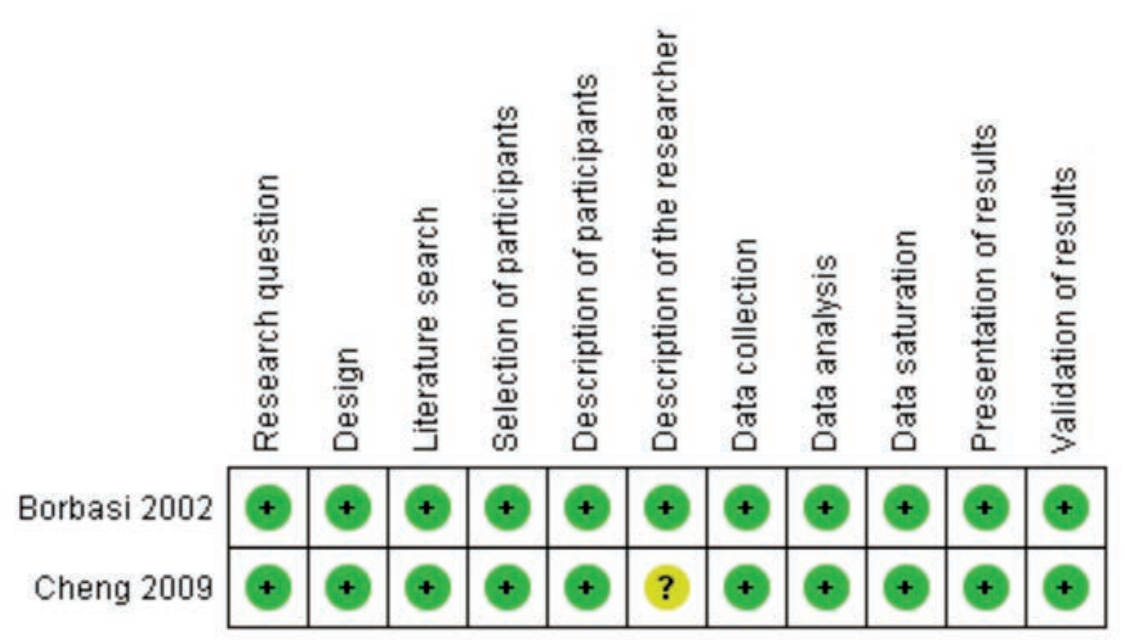

Figure 2. Methodological quality summary.

Table 1. Search strategy.

\begin{tabular}{|c|c|c|}
\hline S13 & (S12) AND (S9) & 67 \\
\hline S12 & (S10) AND (S11) & 738 \\
\hline S11 & (S7) OR (S8) & 194,195 \\
\hline S10 & (S5) OR (S6) & 1223 \\
\hline S9 & $(((\mathrm{S} 1)$ OR (S2)) OR (S3)) OR (S4) & 396,269 \\
\hline S8 & neoplasm & 41,817 \\
\hline S7 & cancer & 175,075 \\
\hline S6 & mucositis & 1223 \\
\hline S5 & oral mucositis & 473 \\
\hline S4 & AB focus group* OR TI focus group* OR SU focus group* & 26,701 \\
\hline S3 & AB interview* OR TI interview* OR SU interview* & 169,830 \\
\hline S2 & SU questionnaire* or AB questionnaire* OR TI questionnaire* & 250,097 \\
\hline S1 & Qualitative research OR AB qualitative OR TI Qualitative & 48,167 \\
\hline \multicolumn{3}{|c|}{ MEDLINE } \\
\hline$\# 9$ & 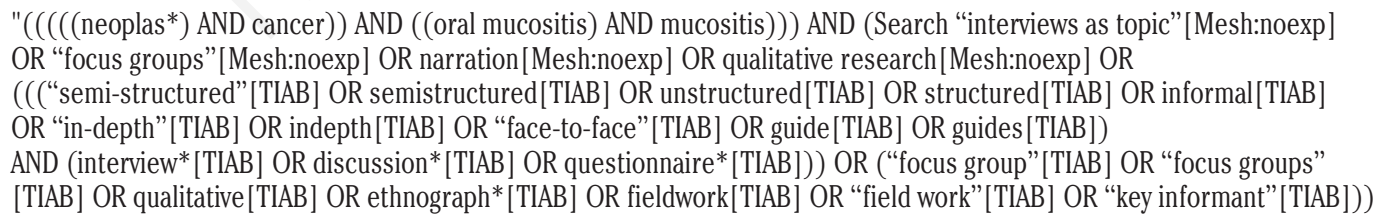 & 20 \\
\hline \#8 & "(((neoplas*) AND cancer)) AND ((oral mucositis) AND mucositis)" & 1579 \\
\hline$\# 7$ & "(neoplas*) AND cancer" & $2,114,430$ \\
\hline$\# 6$ & "(oral mucositis) AND mucositis" & 2934 \\
\hline$\# 5$ & "mucositis" & 6525 \\
\hline$\# 4$ & "oral mucositis" & 25,471 \\
\hline$\# 3$ & "neoplas*" & $2,156,107$ \\
\hline$\# 2$ & "cancer" & 2,907,011 \\
\hline$\# 1$ & $\begin{array}{l}\text { "interviews as topic"[Mesh:noexp] OR "focus groups"[Mesh:noexp] OR narration [Mesh:noexp] OR qualitative research } \\
\text { [Mesh:noexp] OR ((("semi-structured"[TIAB] OR semistructured[TIAB] OR unstructured[TIAB] OR structured[TIAB] } \\
\text { OR informal[TIAB] OR "in-depth"[TIAB] OR indepth[TIAB] OR "face-to-face"[TIAB] OR guide[TIAB] OR guides[TIAB]) } \\
\text { AND (interview*[TIAB] OR discussion*[TIAB] OR questionnaire*[TIAB])) OR ("focus group"[TIAB] OR "focus groups" } \\
\text { [TIAB] OR qualitative[TIAB] OR ethnograph*[TIAB] OR fieldwork[TIAB] OR "field work"[TIAB] OR "key informant"[TIAB])) }\end{array}$ & 211,080 \\
\hline
\end{tabular}


being of patients and their relatives. It causes serious complications with regard to eating, speaking, swallowing, pain, sadness, lack of energy, and distress. Respondents described mucositis as the worst side effect of cancer therapy.

In a widely cited study by Griffin et al..$^{30} 42 \%$ of study participants receiving chemotherapy showed signs of mucositis, which was assessed as one of the most distressing effects of cancer therapy, including hair loss, nausea and loss of appetite. Griffin's study was conducted already in 1993. In the meantime, treatment opportunities concerning side effects like nausea or vomiting significantly improved. So mucositis certainly has another status today.

None of the study participants expected the severity of mucositis and such large restrictions in daily life. This reflects a commonly observed phenomenon in the care of patients with malignant diseases. Immediately after the diagnosis they receive a lot of information about the disease, its treatment and side effects. Shocked by the diagnosis, they cannot integrate all given information. So the scope of side effects will become apparent only when patients begin to suffer from them.

Due to pain and swelling, serious problems with eating, drinking, talking and sleeping may occur. The respondents reported high distress related to eating and drinking which becomes the major problem of their daily life.

Food rejection is alarming for relatives who react with anxiety and put pressure on patients. ${ }^{31}$ From the perspective of relatives, encouragement for eating and drinking expresses that they are worried about the patient's situation. ${ }^{32}$

All study participants were aware how important adequate oral care was to alleviate mucositis-associated pain. Nurses play a significant role in assisting patients to tolerate their treat- ment. Patients valued the range of individual treatments and the nurses' mental support. Nevertheless, study participants expressed their wishes for more emotional support of healthcare professionals.

Surprisingly, we could not identify studies which were conducted in our cultural background. So the findings were discussed with the oncological nurses in the guideline development group regarding transferability. The nurses confirmed the categories identified by the authors of the two studies. Six patient-relevant outcome measures were determined based on this literature review and the experience of the nurses. Patient-relevant outcomes address: pain, prevention of infectious diseases, eating and drinking, xerostomia, sleep disorders, and impaired sense of taste. According to the patient-relevant outcomes 29 clinical questions were formulated, which should be answered by means of the guideline.

Table 2. Reasons of excluded studies.

\begin{tabular}{ll} 
Reference & Reason of exclusion \\
Armstrong and McCaffrey, $2006^{13}$ & Inadequate focus: quality of life \\
Goldberg et al., $2004^{14}$ & Study design does not fit: quantitative research design \\
\hline Cheng, $2007^{15}$ & Study design does not fit: quantitative research design \\
Chen, $2008^{16}$ & Study design does not fit: quantitative research design \\
\hline Dodd et al., $2001^{17}$ & Study design does not fit: quantitative research design \\
\hline Ethier et al., $2012^{18}$ & Study design does not fit: quantitative research design \\
\hline Green et al., $2010^{19}$ & Inadequate focus: eating problems due to nausea and mucositis \\
Miller et al., $2007^{20}$ & Inadequate focus: feasibility and acceptability of an oral care diary \\
\hline Riordain et al., $2011^{21}$ & Study design does not fit: quantitative research design \\
Riordain et al., $2011^{22}$ & Inadequate sample: patients with chronic mucositis independent of cause \\
\hline Rose-Ped et al., $2002^{23}$ & Study design does not fit: quantitative research design \\
Larsson et al., $2003^{24}$ & Inadequate focus: eating problems \\
\hline Syrjala et al., $2004^{25}$ & Study design does not fit: quantitative research design \\
Wong et al., $2006^{26}$ & Study design does not fit: quantitative research design \\
\hline
\end{tabular}

Table 3. Summary of included studies.

\section{Borbasi et al., $2002^{28}$}

Research question/aim

Design

Sample, setting

Data collection

Cheng, $2009^{2 \pi}$

Research question/aim

Design

Sample, setting

Data collection

SD, standard deviation; ALL, acute lymphoblastic leukemia.
What are the experiences with mucositis in patients who are receiving intensive cytotoxic treatment associated with autologous haematopoietic stem cell transplantation? Interpretive phenomenological study Six patients: 4 women, 2 men, age range 38-63 years; metropolitan teaching hospital in South Australia Weekly indepth interviews for 4 weeks and at week 8 , week 12 or until the symptoms of oral mucositis had resolved; interviews: $45 \mathrm{~min}$ to $1 \mathrm{~h}$; total of 19 interviews

Description of children's and their parents' lived experiences of oral mucositis and to explore their needs in relation to oral mucositis

Descriptive phenomenological study

22 pediatric cancer patients and their parents; children: mean age 12.1 years (SD 3.4), 12 males; ALL (n=9), osteosarcoma (n=7); parents: mean age 41.1 years (SD 8.3), 21 (95\%) females; children's cancer center of a regional university hospital in Hong Kong

Semi-structured interviews; children's interviews 20 to 30 min, interviews with parents 30 to 40 min

[page 10]

[Nursing Reports 2014; 4:3647]

OPEN ACCESS 


\section{Study limitations}

The review has some limitations. Due to limitations of financial resources we could not carry out hand-searching, involve an information scientist and use more databases. We tried to find the existing literature using a broad search strategy and checking the reference lists. Therefore existing studies may have almost completely detected. Although few studies have been found, this review can be a basis for further research.

\section{Conclusions}

In order to develop an evidence-based clinical practice guideline for management of tumor therapy-induced oral mucositis, it was necessary to capture the patient's perspective. Oral mucositis caused by cancer therapy is associated with serious complications concerning pain, eating, swallowing, speaking, sadness, lack of energy, and distress. Mucositis was described as the worst side effect of cancer therapy. Patient-relevant outcomes address pain, prevention of infectious diseases, eating and drinking, xerostomia, sleep disorders, and impaired sense of taste. Even if nurses in the guideline development group largely confirmed the results, it is necessary to examine the experience of mucositis in German speaking countries in order to derive meaningful recommendations for health care professionals. It is very resource-consuming to develop guidelines for individual cantons. Therefore a national strategy would be helpful.

\section{References}

1. Eilers J, Million R. Clinical update: prevention and management of oral mucositis in patients with cancer. Oncol Nurs 2011;27: e1-16.

2. Clarkson JE, Worthington HV, Eden OB. Interventions for treating oral mucositis for patients with cancer receiving treatment. Austr Dental J 2008;53:363-5.

3. Sonis ST. Pathobiology of oral mucositis: novel insights and opportunities. J Support Oncol 2007;5:3-11.

4. Worthington HV, Clarkson JE, Bryan G, et al. Interventions for preventing oral mucositis for patients with cancer receiving treatment. Cochrane Database Syst Rev 2011; (4):CD000978.

5. Napenas JJ, Shetty KV, Streckfus CF. Oral mucositis: review of pathogenesis, diagnosis, prevention, and management. General dentistry (Gen Dent) 2007;55:335-44; quiz
$345-6,376$.

6. Grundy M, Ghazi F. Research priorities in haemato-oncology nursing: results of a literature review and a Delphi study. Eur J Oncol Nurs 2009;13:235-49.

7. Ivanovic N, Widmer C, Panfil EM. First development of evidence-based nursing guidelines on selected topics in Switzerland. Guidelines International Network. G-I-N Conference 2012, Berlin. Available from: http:/www.egms.de/static/ en/meetings/gin2012/12gin244.shtml

8. Schunemann HJ, Wiercioch W, Etxeandia I, et al. Guidelines 2.0: systematic development of a comprehensive checklist for a successful guideline enterprise. CMAJ 2014;186:E123-42.

9. University of Texas School of Public Health. Search filters for qualitative studies. Available from: http://libguides. sph.uth. tmc.edu/ovid_medline_filters Accessed: February 12, 2014.

10. Wilczynski NL, Marks S, Haynes RB. Search strategies for identifying qualitative studies in CINAHL. Qual Health Res 2007;17:705-10.

11. Behrens J, Langer G. Evidence-based nursing and caring: Methoden und Ethik der Pflegepraxis und Versorgungsforschung. 3rd ed. Bern: Hans Huber; 2010.

12. Thomas J, Harden A. Methods for the thematic synthesis of qualitative research in systematic reviews. BMC Med Res Methodol 2008;8:45.

13. Armstrong JA, McCaffrey R. The effects of mucositis on quality of life in patients with head and neck cancer. Clin J Oncol Nurs 2006;10:53-6.

14. Goldberg S, Chiang L, Selina N, Hamarman S. Patient perceptions about chemotherapyinduced oral mucositis: implications for primary/secondary prophylaxis strategies. Support Care Cancer 2004; 2004;12:526-30.

15. Cheng KK. Oral mucositis and quality of life of Hong Kong Chinese patients with cancer therapy. Eur J Oncol Nurs 2007;11: 36-42.

16. Chen H. Patients' experiences and perceptions of chemotherapy-induced oral mucositis in a day unit. Cancer Nursing 2008;31:363-9.

17. Dodd MJ, Dibble S, Miaskowski C, et al. A comparison of the affective state and quality of life of chemotherapy patients who do and do not develop chemotherapy-induced oral mucositis. J Pain Symptom Manage 2001;21:498-505.

18. Ethier M, Regier DA, Tomlinson D, et al. Perspectives toward oral mucositis prevention from parents and health care professionals in pediatric cancer. Support Care Cancer 2012;20:1771-7.

19. Green R, Horn H, Erickson JM. Eating Experiences of children and adolescents with chemotherapy-related nausea and mucositis. J Pediatr Oncol Nurs 2010;27: 209-16.

20. Miller M, Taylor A, Kearney N, et al. Evaluation of the feasibility and acceptability of an oral care diary by patients during chemotherapy. Int J Nurs Stud 2007;44: 693-701.

21. Riordain RN, Meaney S, McCreary C. A patient-centered approach to developing a quality-of-life questionnaire for chronic oral mucosal diseases. Oral Surg Oral Med Oral Pathol Oral Radiol Endod 2011;111: 578-86.e2.

22. Riordain RN, Meaney S, McCreary C. Impact of chronic oral mucosal disease on daily life: preliminary observations from a qualitative study. Oral Dis 2011;17:265-9.

23. Rose-Ped AM, Bellm LA, Epstein JB, et al. Complications of radiation therapy for head and neck cancers. The patient's perspective. Cancer Nursing 2002;25:461-7; quiz 468-9.

24. Larsson M, Hedelin B, Athlin E. Lived experiences of eating problems for patients with head and neck cancer during radiotherapy. J Clin Nurs 2003;12:562-70.

25. Syrjala KL, Hays RD, Kallich JD, et al. Impact of oral mucositis and its sequelae on quality of life. J Support Oncol 2004;2:79-80.

26. Wong PC, Dodd MJ, Miaskowski C, et al. Mucositis pain induced by radiation therapy: prevalence, severity, and use of self-care behaviors. J Pain Symptom Manage 2006; 32:27-37.

27. Cheng KK. Oral mucositis: a phenomenological study of pediatric patients' and their parents' perspectives and experiences. Support Care Cancer 2009;17:829-37.

28. Borbasi S, Cameron K, Quested B, et al. More than a sore mouth: patients' experience of oral mucositis. Oncol Nurs Forum 2002;29:1051-7.

29. Nicolatou-Galitis 0, Sarri T, Bowen J, et al. Systematic review of amifostine for the management of oral mucositis in cancer patients. Support Care Cancer 2013;21: 35764.

30. Griffin AM, Butow PN, Coates AS, et al. On the receiving end. V: Patient perceptions of the side effects of cancer chemotherapy in 1993. Ann Oncol 1996;7:189-95.

31. Reid J, McKenna H, Fitzsimons D, McCance T. Fighting over food: patient and family understanding of cancer cachexia. Oncol Nurs Forum 2009;36:439-45.

32. Hughes N, Neal RD. Adults with terminal illness: a literature review of their needs and wishes for food. J Adv Nurs 2000;32: 1101-7. 